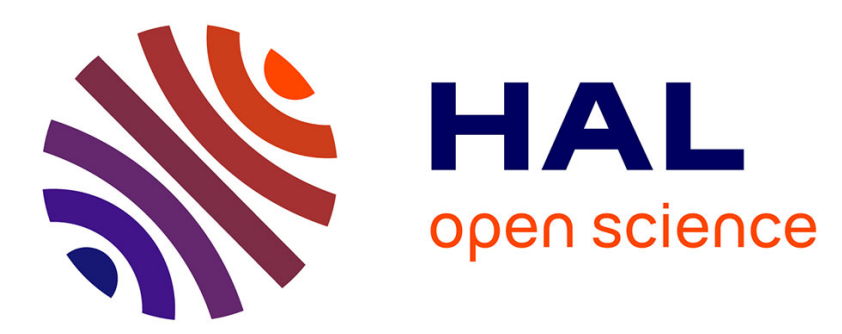

\title{
On convergence conditions for generalized Persidskii systems
}

Wenjie Mei, Denis Efimov, Rosane Ushirobira, Alexander Aleksandrov

\section{To cite this version:}

Wenjie Mei, Denis Efimov, Rosane Ushirobira, Alexander Aleksandrov. On convergence conditions for generalized Persidskii systems. International Journal of Robust and Nonlinear Control, In press. hal-03495478

\section{HAL Id: hal-03495478 \\ https://hal.inria.fr/hal-03495478}

Submitted on 20 Dec 2021

HAL is a multi-disciplinary open access archive for the deposit and dissemination of scientific research documents, whether they are published or not. The documents may come from teaching and research institutions in France or abroad, or from public or private research centers.
L'archive ouverte pluridisciplinaire HAL, est destinée au dépôt et à la diffusion de documents scientifiques de niveau recherche, publiés ou non, émanant des établissements d'enseignement et de recherche français ou étrangers, des laboratoires publics ou privés. 
DOI: $\mathrm{xxx} / \mathrm{xxxx}$

\title{
RESEARCH ARTICLE
}

\section{On convergence conditions for generalized Persidskii systems}

\author{
Wenjie Mei*1 $^{*}$ Denis Efimov ${ }^{1,2}$ | Rosane Ushirobira ${ }^{1}$ | Alexander Aleksandrov ${ }^{3}$
}

${ }^{1}$ Inria, Univ. Lille, CNRS, UMR 9189 CRIStAL, Lille, France

${ }^{2}$ ITMO University, Saint Petersburg, Russia

${ }^{3}$ Saint Petersburg State University, Saint

Petersburg, Russia

\section{Correspondence}

*Wenjie Mei, Inria, Univ. Lille, CNRS, UMR

9189 - CRIStAL, F-59000 Lille, France.

Email: Wenjie.Mei@inria.fr

\begin{abstract}
Summary
The convergence conditions for a class of generalized Persidskii systems and their discretized dynamics are introduced, which can be checked through linear (matrix) inequalities. The case of almost periodic convergence for this class of systems with almost periodic input is also studied. The proposed results are applied to a LotkaVolterra model and opinion dynamics.
\end{abstract}

\section{KEYWORDS:}

convergence conditions, generalized Persidskii systems, LMI

\section{1 | INTRODUCTION}

A dynamical system is convergent if it admits a unique and bounded asymptotically stable solution ${ }^{12}$. So computing only one trajectory is sufficient to determine the asymptotic behavior of all others. This property is similar to the incremental stability 3 (without the boundedness requirement) and the contraction theory is frequently used for its analysis ${ }^{5}$. The convergence property has received increased attention due to its usefulness in system analysis and design, including the case of several co-existing

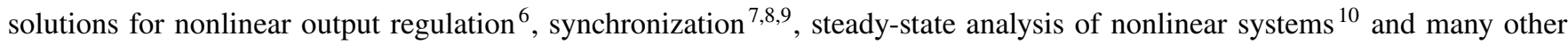
control problems.

It is evident that not all nonlinear systems are convergent: the trajectories may be convergent for some inputs (e.g., zero input), and an example of such a behavior is demonstrated by Duffing's model ${ }^{111}$, in which multiple limit cycles phenomena and subharmonic oscillations may occur. Thus, for nonlinear systems, it is required to consider additional conditions for investigating the convergence property.

We may additionally ask for preservation conditions for the attracting solution of the (almost) periodicity of the input ${ }^{12 / 13}$. Note that in nonlinear systems, the frequency of entertainment is not necessarily kept by the output ${ }^{14}$, and a typical example is again the Duffing's model, which can generate chaotic trajectories under harmonic excitation. The relations between the existence of almost periodic solutions and properties of the right-hand sides of dynamical systems were intensively studied during the last century (see, e.g., Markov's theorem ${ }^{15}$ ).

The convergence property itself also has a rich history of investigation. For example, Pliss ${ }^{16}$ studied convergence of the system $\dot{x}=f(t, x)$ with the periodic right-hand side. Later, Demidovich ${ }^{17}$ extended the particular case analyzed by Pliss to a more general $f$. In the same vein, Zubov $\underline{18119}$ established a criterion of periodic/almost periodic convergence for nonlinear systems. Further exploration of convergence properties for nonlinear systems includes convergence-like properties by Lyapunov's second method ${ }^{20}$, sufficient conditions for the convergence of a system with a scalar nonlinearity satisfying an incremental sector condition $^{21}$, the convergence of perturbed nonlinear systems $s^{2213}$, and convergence conditions for a class of nonlinear dynamics ${ }^{23}$.

Digital revolution highlighted importance and originated discrete-time modeling for many phenomena. In addition, for realworld systems having the models formulated in continuous time, for implementation or simulation, these models have to be discretized. For strongly nonlinear systems the obtained discrete-time representations may loose the stability and convergence properties of the continuous-time counterparts ${ }^{2425}$. This motivates development of conditions of similar quality performance characteristics for the systems functioning in discrete time. 
In this paper, the convergence property and existence of almost periodic trajectories will be studied in continuous and discrete time for a class of Persidskii systems, which was introduced in ${ }^{26 / 27}$ as $\dot{x}=A_{0} x+A_{1} f(x)$, and recently its generalized version was investigated in ${ }^{2829}$ by taking into account multiple nonlinearities: $\dot{x}=A_{0} x+\sum_{i=1}^{M} A_{i} f_{i}(x)$ (more detailed definition is given below). The advantage of this class of models is that despite its important nonlinear nature (with $M \geq 1$ ), a Lyapunov function is suggested in the literature such that the analysis of its properties can be performed by solving linear matrix inequalities (LMIs), which is a constructive approach.

The organization of this paper is as follows. In Section 2 the preliminaries and the definitions of used stability properties are presented. The class of considered systems and the imposed conditions for nonlinearities are introduced in Section 3 The basic conditions of convergence for generalized Persidskii systems, and of almost periodic convergence for those systems with Metzler coefficient matrices are given in Section 4 In Section 5 convergence conditions for two types of discretizations of the considered systems are studied. The Lotka-Volterra model and opinion dynamics are considered to illustrate the efficiency of the proposed results in Section 6

\section{NOTATION}

- $\mathbb{N}, \mathbb{Z}$ and $\mathbb{R}$ represent the sets of natural numbers, integers and real numbers, respectively, $\mathbb{R}_{+}=\{s \in \mathbb{R}: s \geq 0\}$. $\mathbb{R}^{n}$ $\left(\mathbb{R}_{+}^{n}\right)$ and $\mathbb{R}^{m \times n}$ denote the vector spaces of $n$-tuples of real numbers (nonnegative real numbers) and $m \times n$ real matrices, respectively. The set of $n \times n$ diagonal matrices with nonnegative elements on the diagonal is denoted by $\mathbb{D}_{+}^{n}$.

- The symbol $\|\cdot\|$ refers to the Euclidean norm on $\mathbb{R}^{n}$ (and the induced matrix norm $\|A\|$ for a matrix $A \in \mathbb{R}^{m \times n}$ ).

- The transpose of a matrix $A \in \mathbb{R}^{n \times n}$ is denoted by $A^{\top}$.

- For $p, n \in \mathbb{N}$ with $p \leq n$, the notation $\overline{p, n}$ is used to represent the set $\{p, \ldots, n\}$. For all $i, j \in \overline{p, n}$, let $\left(B_{i, j}\right)_{i, j=p}^{n}$ denote the block matrix $\left[\begin{array}{ccc}B_{p, p} & \cdots & B_{p, n} \\ \vdots & \ddots & \vdots \\ B_{n, p} & \cdots & B_{n, n}\end{array}\right]$.

- The $p \times n$ block diagonal matrix with matrices $v_{i} \in \mathbb{R}^{p_{i} \times n_{i}}\left(p=\sum_{i=1}^{N} p_{i}, n=\sum_{i=1}^{N} n_{i}\right)$ along the main diagonal is denoted by $\operatorname{diag}\left(v_{1}, \ldots, v_{N}\right)$. Let $I_{n}$ stand for the $n \times n$ identity matrix, and denote the vector of dimension $n$ with all elements equal 1 by $\mathbf{1}_{n}$.

- For a vector $v \in \mathbb{R}^{n}, v>0(v<0)$ represents $v_{i}>0\left(v_{i}<0\right)$ for $i \in \overline{1, n}$. For $r \in \mathbb{R}$, let $|r|$ denote its absolute value.

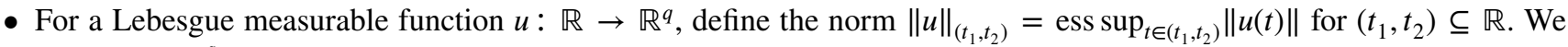
denote by $\mathscr{L}_{\infty}^{q}$ the space of functions $u$ with $\|u\|_{\infty}:=\|u\|_{(-\infty,+\infty)}<+\infty$. For a bounded input sequence $\{\tilde{u}(k)\}_{k \in \mathbb{Z}}$, $\tilde{u}(k) \in \mathbb{R}^{q}$, define the norm $\|\tilde{u}\|_{\overline{k_{1}, k_{2}}}=\sup _{k^{\prime} \in \overline{k_{1}, k_{2}}}\left\|\tilde{u}\left(k^{\prime}\right)\right\|$ for $k_{1}, k_{2} \in \mathbb{Z}$. Let $\mathcal{U}_{\infty}^{q}$ denote the space of functions $\tilde{u}$ with $\|\tilde{u}\|_{\infty}:=\|\tilde{u}\|_{k_{1}, k_{2}}<+\infty$ for $k_{1} \rightarrow-\infty, k_{2} \rightarrow+\infty$.

- A continuous function $\sigma: \mathbb{R}_{+} \rightarrow \mathbb{R}_{+}$belongs to class $\mathscr{K}$ if it is strictly increasing and $\sigma(0)=0$; it belongs to class $\mathscr{K}_{\infty}$ if it is also unbounded. A continuous function $\beta: \mathbb{R}_{+} \times \mathbb{R}_{+} \rightarrow \mathbb{R}_{+}$belongs to class $\mathscr{K} \mathscr{L}$ if $\beta(\cdot, r) \in \mathscr{K}$ and $\beta(r, \cdot)$ is a decreasing to zero function for any fixed $r>0$.

- For a continuously differentiable function $V: \mathbb{R}^{n} \rightarrow \mathbb{R}$, denote by $\nabla V(v) f(v)$ the Lie derivative of $V$ along the vector field $f$ evaluated at point $v \in \mathbb{R}^{n}$.

\section{2 | PRELIMINARIES}

Definition 1. The matrix $A \in \mathbb{R}^{n \times n}$ is said to be Hurwitz if all its eigenvalues lie in the open left half of the complex plane.

Definition 2. The matrix $A \in \mathbb{R}^{n \times n}$ is Metzler if all its off-diagonal entries are nonnegative.

Proposition $1\left({ }^{(30}\right)$. Let $A \in \mathbb{R}^{n \times n}$ be Metzler. Then $A$ is Hurwitz if and only if there exists a vector $v>0$ in $\mathbb{R}^{n}$ with $A^{\top} v<0$. 
Definition $3\left(\frac{15}{15}\right)$. A continuous function $\psi: \mathbb{R} \rightarrow \mathbb{R}^{n}$ is called almost periodic if, for every $\varepsilon>0$ there exists $\tau_{\varepsilon}>0$ such that every interval $\left[r, r+\tau_{\epsilon}\right]$ for any $r \in \mathbb{R}$ contains at least one number $\eta$ for which

$$
\|\psi(t+\eta)-\psi(t)\|<\varepsilon
$$

for all $t \in \mathbb{R}$.

\section{1 | Stability properties in continuous time}

Let the differential equation

$$
\begin{aligned}
& \dot{x}(t)=F(x(t), u(t)), t \geq t_{0} \in \mathbb{R}, \\
& y(t)=H(x(t))
\end{aligned}
$$

be given, where $x(t) \in \mathbb{R}^{n}$ is the state vector; $u(t) \in \mathbb{R}^{q}$ is the external input, $u \in \mathscr{L}_{\infty}^{q} ; y(t) \in \mathbb{R}^{p}$ is the output vector. Moreover, $F: \mathbb{R}^{n+q} \rightarrow \mathbb{R}^{n}$ is a nonlinear continuous mapping and $H: \mathbb{R}^{n} \rightarrow \mathbb{R}^{p}$ is continuously differentiable. For an initial state $x_{0} \in \mathbb{R}^{n}$, initial time $t_{0} \in \mathbb{R}$ and $u \in \mathscr{L}_{\infty}^{q}$ we denote a corresponding solution of the system (1) by $x\left(t, x_{0}, t_{0}, u\right)$ for the values of $t \geq t_{0}$ the solution exists, so the corresponding output is $y\left(t, x_{0}, t_{0}, u\right)=H\left(x\left(t, x_{0}, t_{0}, u\right)\right)$. In the case that $t_{0}=0\left(\right.$ i.e., $\left.t \in \mathbb{R}_{+}\right)$, we simplify the corresponding denotations to: $x\left(t, x_{0}, u\right)$ and $y\left(t, x_{0}, u\right)$, which are also applicable in the sequel.

Definition $4\left(\frac{31}{13}\right)$. The system (1) is called forward complete if for all $x_{0} \in \mathbb{R}^{n}$ and $u \in \mathscr{L}_{\infty}^{q}$, the solution $x\left(t, x_{0}, u\right)$ is uniquely defined for all $t \in \mathbb{R}_{+}$.

Definition $5(\underline{31})$. A forward complete system (1) is said to be

1. input-to-output stable (IOS) if there exist $\beta \in \mathscr{K} \mathscr{L}$ and $\gamma \in \mathscr{K}$ such that

$$
\left\|y\left(t, x_{0}, u\right)\right\| \leq \beta\left(\left\|x_{0}\right\|, t\right)+\gamma\left(\|u\|_{\infty}\right), \forall t \in \mathbb{R}_{+}
$$

for any $x_{0} \in \mathbb{R}^{n}$ and $u \in \mathscr{L}_{\infty}^{q}$. In the special case when $y=x$, the IOS property is called input-to-state stability (ISS).

2. state-independent input-to-output stable (SIIOS) if there exist $\beta \in \mathscr{K} \mathscr{L}, \gamma \in \mathscr{K}$ such that

$$
\left\|y\left(t, x_{0}, u\right)\right\| \leq \beta\left(\left\|H\left(x_{0}\right)\right\|, t\right)+\gamma\left(\|u\|_{\infty}\right), \forall t \in \mathbb{R}_{+}
$$

for any $x_{0} \in \mathbb{R}^{n}$ and $u \in \mathscr{L}_{\infty}^{q}$.

3. robustly output stable (ROS) if there exist a smooth function $\alpha \in \mathscr{K}_{\infty}$ and $\beta \in \mathscr{K} \mathscr{L}$ such that the system

$$
\dot{x}=\tilde{F}(x, \kappa):=F(x, \kappa \alpha(\|H(x)\|))
$$

is forward complete, and the estimate

$$
\left\|y_{\alpha}\left(t, x_{0}, \kappa\right)\right\| \leq \beta\left(\left\|x_{0}\right\|, t\right), \forall t \in \mathbb{R}_{+}
$$

is satisfied for all $x_{0} \in \mathbb{R}^{n}, \kappa \in \mathscr{L}_{\infty}^{q}$ with $\|\kappa\|_{\infty} \leq 1$ and $y_{\alpha}\left(t, x_{0}, \kappa\right)=H\left(x\left(t, x_{0}, \kappa\right)\right)$ denotes the output function of the system (2).

Definition 6 ( $(31)$. A forward complete system (1) is said to be uniformly bounded-input-bounded-state stable (UBIBS) if there exists $\beta \in \mathscr{K}$ such that

for all $x_{0} \in \mathbb{R}^{n}$ and $u \in \mathscr{L}_{\infty}^{q}$.

$$
\left\|x\left(t, x_{0}, u\right)\right\| \leq \max \left\{\beta\left(\left\|x_{0}\right\|\right), \beta\left(\|u\|_{\infty}\right)\right\}, \forall t \in \mathbb{R}_{+}
$$

\section{2 | Convergence definitions}

Definition 7 ( 1 ). The system (1) is convergent if it admits a unique bounded solution for $t \in \mathbb{R}$ that is globally asymptotically stable.

Definition 8 (19). The system (1) possesses almost periodic convergence property, if it is convergent and the admitted solution is almost periodic. 


\subsection{Stability properties in discrete time}

Consider a discrete-time system:

$$
\begin{aligned}
x(k+1) & =\bar{F}(x(k), u(k)), \\
y(k) & =H(x(k)),
\end{aligned}
$$

where $x(k) \in \mathbb{R}^{n}, u(k) \in \mathbb{R}^{q}$ and $y(k) \in \mathbb{R}^{p}$ are defined as before; $k_{0} \leq k \in \mathbb{Z}$ with $k_{0} \in \mathbb{Z} ; \bar{F}: \mathbb{R}^{n+q} \rightarrow \mathbb{R}^{n}$ is a continuous mapping; $u \in \mathscr{U}_{\infty}^{q}$, the mapping $H$ and the solution for (3) are defined as in the system (1).

Definition 9 (32). The system (3) is called input-to-state stable (ISS) if there exist $\beta \in \mathscr{K} \mathscr{L}$ and $\gamma \in \mathscr{K}$ such that

$$
\left\|x\left(k, x_{0}, u\right)\right\| \leq \beta\left(\left\|x_{0}\right\|, k\right)+\gamma\left(\|u\|_{\infty}\right)
$$

for any $k \in \mathbb{N}, x_{0} \in \mathbb{R}^{n}$ and $u \in \mathcal{U}_{\infty}^{q}$.

\section{4 | Lyapunov characterizations}

Definition 10 (31). For the system (11), a smooth function $V: \mathbb{R}^{n} \rightarrow \mathbb{R}_{+}$is

1. an ISS-Lyapunov function if there exist $\alpha_{1}, \alpha_{2}, \alpha_{3} \in \mathscr{K}_{\infty}$ and $\chi \in \mathscr{K}$ such that

$$
\begin{gathered}
\alpha_{1}(\|x\|) \leq V(x) \leq \alpha_{2}(\|x\|), \\
\|x\| \geq \chi(\|u\|) \Rightarrow \nabla V(x) F(x, u) \leq-\alpha_{3}(\|x\|)
\end{gathered}
$$

for all $x \in \mathbb{R}^{n}$ and $u \in \mathbb{R}^{q}$.

2. an IOS-Lyapunov function if there exist $\alpha_{1}, \alpha_{2} \in \mathscr{K}_{\infty}, \chi \in \mathscr{K}$ and $\alpha_{3} \in \mathscr{K} \mathscr{L}$ such that

$$
\begin{gathered}
\alpha_{1}(\|H(x)\|) \leq V(x) \leq \alpha_{2}(\|x\|), \\
V(x) \geq \chi(\|u\|) \Rightarrow \nabla V(x) F(x, u) \leq-\alpha_{3}(V(x),\|x\|)
\end{gathered}
$$

for all $x \in \mathbb{R}^{n}$ and $u \in \mathbb{R}^{q}$.

3. an SIIOS-Lyapunov function if there exist $\alpha_{1}, \alpha_{2} \in \mathscr{K}_{\infty}$ and $\chi, \alpha_{3} \in \mathscr{K}$ such that

$$
\begin{gathered}
\alpha_{1}(\|H(x)\|) \leq V(x) \leq \alpha_{2}(\|H(x)\|), \\
V(x) \geq \chi(\|u\|) \Rightarrow \nabla V(x) F(x, u) \leq-\alpha_{3}(V(x))
\end{gathered}
$$

for all $x \in \mathbb{R}^{n}$ and $u \in \mathbb{R}^{q}$.

4. a ROS-Lyapunov function if there exist $\alpha_{1}, \alpha_{2} \in \mathscr{K}_{\infty}, \chi \in \mathscr{K}$ and $\alpha_{3} \in \mathscr{K} \mathscr{L}$ such that

$$
\begin{gathered}
\alpha_{1}(\|H(x)\|) \leq V(x) \leq \alpha_{2}(\|x\|), \\
\|H(x)\| \geq \chi(\|u\|) \Rightarrow \nabla V(x) F(x, u) \leq-\alpha_{3}(V(x),\|x\|)
\end{gathered}
$$

for all $x \in \mathbb{R}^{n}$ and $u \in \mathbb{R}^{q}$.

Definition 11 (32). For the system (3), a smooth function $V: \mathbb{R}^{n} \rightarrow \mathbb{R}_{+}$is an ISS-Lyapunov function if there exist $\alpha_{1}, \alpha_{2}, \alpha_{3} \in$ $\mathscr{K}_{\infty}$ and $\chi \in \mathscr{K}$ such that

$$
\begin{gathered}
\alpha_{1}(\|x\|) \leq V(x) \leq \alpha_{2}(\|x\|), \\
\|x\| \geq \chi(\|u\|) \Rightarrow V(\bar{F}(x, u))-V(x) \leq-\alpha_{3}(\|x\|)
\end{gathered}
$$

for all $x \in \mathbb{R}^{n}$ and $u \in \mathbb{R}^{q}$.

Theorem 1 (33/32). The system (1) or (3) is ISS if and only if it admits an ISS-Lyapunov function.

Theorem 2 (31). A UBIBS system (1) is IOS (ROS) if and only if it admits an IOS (ROS)-Lyapunov function.

Theorem $3\left(\frac{31}{11}\right)$. A forward complete system (1) is SIIOS if and only if it admits an SIIOS-Lyapunov function. 
Remark 1. The UBIBS requirement can be dropped in the sufficient conditions of IOS or ROS if the system (1) is forward complete (or it possesses the unboundedness observability property ${ }^{34}$ ) and admits an IOS-Lyapunov function satisfying the conditions (5), with

$$
V(x) \geq \chi(\|u\|) \Rightarrow \nabla V(x) F(x, u) \leq-\alpha_{3}(V(x))
$$

or a ROS-Lyapunov function with the conditions (7), and

$$
\|H(x)\| \geq \chi(\|u\|) \Rightarrow \nabla V(x) F(x, u) \leq-\alpha_{3}(V(x))
$$

for all $x \in \mathbb{R}^{n}$ and $u \in \mathbb{R}^{q}$, some $\alpha_{1}, \alpha_{2} \in \mathscr{K}_{\infty}$ and $\chi, \alpha_{3} \in \mathscr{K}$, respectively.

In the rest of the paper, to lighten the notation the time-dependency of variables might remain implicitly understood, for instance we will write $x$ for $x(t)$ or $x(k)$.

\section{3 | PROBLEM STATEMENT}

Consider the following system in generalized Persidskii form 27 :

$$
\dot{x}(t)=A_{0} x(t)+\sum_{j=1}^{M} A_{j} F_{j}(x(t))+\varphi(t), t \geq t_{0},
$$

where $x(t) \in \mathbb{R}^{n}$ is the state vector; $t_{0} \in \mathbb{R}$ denotes the initial time and $x\left(t_{0}\right)=x_{0} ; A_{s} \in \mathbb{R}^{n \times n}$ for $s \in \overline{0, M}(M \in \mathbb{N} \backslash\{0\})$; $F_{j}(x(t))=\left[f_{j}^{1}\left(x_{1}(t)\right) \ldots f_{j}^{n}\left(x_{n}(t)\right)\right]^{\top}$ for $j \in \overline{1, M}$ are the functions ensuring the existence of the solutions of the system (11) at least locally in time; the disturbance input $\varphi=\left[\begin{array}{lll}\varphi_{1} & \ldots & \varphi_{n}\end{array}\right]^{\top} \in \mathscr{L}_{\infty}^{n}$.

In this paper, the corresponding term (in sum or a sequence) must be omitted if for an index the upper bound is smaller than the lower one.

The sector restrictions on $f_{j}^{i}: \mathbb{R} \rightarrow \mathbb{R}, j \in \overline{1, M}, i \in \overline{1, n}$, are imposed as:

Assumption 1. Assume that for any $i \in \overline{1, n}$ and $j \in \overline{1, M}$,

$$
v f_{j}^{i}(v)>0, \forall v \in \mathbb{R} \backslash\{0\} .
$$

Under Assumption 1 there exists an index $m \in \overline{0, M}$ such that for all $i \in \overline{1, n}, a \in \overline{1, m}$

$$
\lim _{v \rightarrow \pm \infty} f_{a}^{i}(v)= \pm \infty
$$

and there exists $\mu \in \overline{m, M}$ such that for all $i \in \overline{1, n}, b \in \overline{1, \mu}$

$$
\lim _{v \rightarrow \pm \infty} \int_{0}^{v} f_{b}^{i}(r) d r=+\infty
$$

In such a case $m=0$ implies that all nonlinearities are bounded (at least in positive or negative direction for a component of the state vector).

Assumption 2. Assume that the functions $f_{j}^{i}$ are continuous and strictly increasing for all $i \in \overline{1, n}$ and $j \in \overline{1, M}$.

Note that $\mu=M$ under Assumption 2

Along with (11), consider the system

$$
\dot{y}(t)=A_{0} y(t)+\sum_{j=1}^{M} A_{j} F_{j}(y(t))+\varphi(t)
$$

with the same input but with different initial conditions $y\left(t_{0}\right) \in \mathbb{R}^{n}$. Define $z=y-x$, then the error dynamics of 111 , (12) is

$$
\dot{z}=A_{0} z+\sum_{j=1}^{M} A_{j} G_{j}(x, z),
$$


where

$$
\begin{aligned}
G_{j}(x, z) & =F_{j}(z+x)-F_{j}(x)=\left[g_{j}^{1}\left(x_{1}, z_{1}\right) \ldots g_{j}^{n}\left(x_{n}, z_{n}\right)\right]^{\top} \in \mathbb{R}^{n}, \\
g_{j}^{r}\left(x_{r}, z_{r}\right) & :=f_{j}^{r}\left(z_{r}+x_{r}\right)-f_{j}^{r}\left(x_{r}\right), \forall j \in \overline{1, M}, r \in \overline{1, n} .
\end{aligned}
$$

Note that for any $x \in \mathbb{R}^{n}$ the functions $G_{j}$ in the variable $z \in \mathbb{R}^{n}$ satisfy the properties formulated in assumptions 1 and 2

We then impose the following assumption used for the stability analysis of the system $(13)$ in Section 4 .

Assumption 3. Assume that there exist $S_{0}^{j}, S_{1}^{j}, S_{2}^{j}, S_{3}^{j, j^{\prime}}, H_{0}^{j}, H_{1}^{j}, H_{2}^{j}, H_{3}^{j, j^{\prime}} \in \mathbb{D}_{+}^{n}$ with $j, j^{\prime} \in \overline{1, M}$ such that

$$
G_{j}(x, z)^{\top} G_{j}(x, z) \leq z^{\top} S_{0}^{j} z+2 z^{\top} S_{1}^{j} G_{j}(x, z)+2 z^{\top} S_{2}^{j} F_{j}(z)+2 \sum_{j^{\prime}=1}^{M} G_{j}(x, z)^{\top} S_{3}^{j, j^{\prime}} F_{j^{\prime}}(z)
$$

and

$$
F_{j}(z)^{\top} F_{j}(z) \leq z^{\top} H_{0}^{j} z+2 z^{\top} H_{1}^{j} G_{j}(x, z)+2 z^{\top} H_{2}^{j} F_{j}(z)+2 \sum_{j^{\prime}=1}^{M} G_{j}(x, z)^{\top} H_{3}^{j, j^{\prime}} F_{j^{\prime}}(z)
$$

for all $x, y \in \mathbb{R}^{n}$ and $z=x-y$.

Any globally Lipschitz function $F_{j}$ satisfies such a condition (moreover, in our setting, the local Lipschitz continuity is enough, as it will be explained in Remark 2 below).

Note that for analysis of the convergence property in (11) we need to consider the system with $t \in \mathbb{R}$. However, the time dependence of the right-hand side of (11) comes through the input $\varphi(t)$ only, then using IOS/ISS framework we can investigate the global stability of that system for $t \in \mathbb{R}_{+}$with $\varphi \in \mathcal{L}_{\infty}^{n}$, which allows us next to extend the obtained results to $t \in \mathbb{R}$.

\section{4 | MAIN RESULTS}

The conditions of (almost periodic) convergence of (11) are given in this section for the general case and for the case of positive systems (all matrices $A_{s}, s \in \overline{0, M}$ are Metzler).

\section{1 | General convergence conditions}

Theorem 4. Let assumptions 1,2 and 3 be satisfied. If there exist $0 \leq P=P_{\tilde{\Lambda}^{\top}}, \tilde{P}=\tilde{P}^{\top} \in \mathbb{R}^{n \times n} ;\left\{\Lambda^{j}=\right.$ $\left.\operatorname{diag}\left(\Lambda^{j, 1}, \ldots, \Lambda^{j, n}\right)\right\}_{j=1}^{M}, \quad\left\{\tilde{\Lambda}^{j}=\operatorname{diag}\left(\tilde{\Lambda}^{j,}, \ldots, \tilde{\Lambda}^{j, n}\right)\right\}_{j=1}^{M}, \quad\left\{\Xi^{s}\right\}_{s=0}^{M}, \quad\left\{\Upsilon_{s, r}\right\}_{0 \leq s<r \leq M},\left\{\tilde{\Upsilon}_{j, j^{\prime}}\right\}_{j, j^{\prime}=1}^{M}, \quad\left\{\Gamma_{j}\right\}_{j=1}^{M}, \quad\left\{\Omega_{j}\right\}_{j=1}^{M} \subset \mathbb{D}_{+}^{n} ;$ $\tilde{\Xi}^{0} \in \mathbb{D}_{+}^{n} ; 0<\Phi=\Phi^{\top} \in \mathbb{R}^{n \times n} ; \gamma, \theta>0$ such that

$$
\begin{gathered}
P+\sum_{j=1}^{\mu} \Lambda^{j}>0 ; Q=Q^{\top} \leq 0, \\
\tilde{P}+\sum_{j=1}^{\mu} \tilde{\Lambda}^{j}>0 ; \tilde{Q}=\tilde{Q}^{\top} \leq 0, \\
\Xi^{0}+\sum_{j=1}^{M} \Upsilon_{0, j}+\sum_{s=1}^{m} \Xi^{s}+\sum_{s=1}^{m} \sum_{r=s+1}^{m} \Upsilon_{s, r}>0, \\
\tilde{\Xi}^{0}-\gamma \sum_{j=1}^{M} S_{0}^{j}-\theta \sum_{j=1}^{M} H_{0}^{j} \geq 0, \Gamma_{j}-\gamma S_{1}^{j}-\theta H_{1}^{j} \geq 0, \Omega_{j}-\gamma S_{2}^{j}-\theta H_{2}^{j} \geq 0, \tilde{\Upsilon}_{j, j^{\prime}}-\gamma S_{3}^{j, j^{\prime}}-\theta H_{3}^{j, j^{\prime}} \geq 0, \\
\tilde{\Xi}^{0}-\gamma \sum_{j=1}^{M} S_{0}^{j}-\theta \sum_{j=1}^{M} H_{0}^{j}+\sum_{j=1}^{M}\left(\Gamma_{j}-\gamma S_{1}^{j}-\theta H_{1}^{j}+\Omega_{j}-\gamma S_{2}^{j}-\theta H_{2}^{j}\right)+\sum_{j=1}^{M} \sum_{j^{\prime}=1}^{M} \tilde{\Upsilon}_{j, j^{\prime}}-\gamma S_{3}^{j, j^{\prime}}-\theta H_{3}^{j, j^{\prime}}>0,
\end{gathered}
$$

where

$$
Q=\left(Q_{a, b}\right)_{a, b=1}^{M+2}, \tilde{Q}=\left(\tilde{Q}_{a, b}\right)_{a, b=1}^{3}
$$


with

$$
\begin{gathered}
Q_{1,1}=A_{0}^{\top} P+P A_{0}+\Xi^{0}, \\
Q_{j+1, j+1}=A_{j}^{\top} \Lambda^{j}+\Lambda^{j} A_{j}+\Xi^{j}, j \in \overline{1, M}, \\
Q_{1, j+1}=P A_{j}+A_{0}^{\top} \Lambda^{j}+\Upsilon_{0, j}, j \in \overline{1, M}, \\
Q_{s+1, r+1}=A_{s}^{\top} \Lambda^{r}+\Lambda^{s} A_{r}+\Upsilon_{s, r}, s \in \overline{1, M-1}, r \in \overline{s+1, M}, \\
Q_{1, M+2}=P, Q_{M+2, M+2}=-\Phi, Q_{j+1, M+2}=\Lambda^{j}, j \in \overline{1, M}, \\
\tilde{Q}_{1,1}=A_{0}^{\top} P+P A_{0}+\tilde{\Xi}^{0}, \tilde{Q}_{2,2}=-\gamma I_{n M}, \tilde{Q}_{1,2}=P A+\Gamma, \\
\tilde{Q}_{1,3}=A_{0}^{\top} \tilde{\Lambda}+\Omega, \tilde{Q}_{2,3}=A^{\top} \tilde{\Lambda}+\tilde{\Upsilon}, \tilde{Q}_{3,3}=-\theta I_{n M}, \\
A=\left[A_{1} \ldots A_{M}\right], \Gamma=\left[\Gamma_{1} \ldots \Gamma_{M}\right], \\
\tilde{\Lambda}=\left[\tilde{\Lambda}^{1} \ldots \tilde{\Lambda}^{M}\right], \Omega=\left[\Omega_{1} \ldots \Omega_{M}\right], \tilde{\Upsilon}=\left(\tilde{\Upsilon}_{j, j^{\prime}}\right)_{j, j^{\prime}=1}^{M},
\end{gathered}
$$

then a forward complete system $(11)$ is convergent.

Proof. Consider a candidate Lyapunov function

$$
V(x)=x^{\top} P x+2 \sum_{j=1}^{M} \sum_{i=1}^{n} \Lambda^{j, i} \int_{0}^{x_{i}} f_{j}^{i}(\tau) d \tau,
$$

which is positive definite and radially unbounded due to Finsler's lemma under the condition (14) and Assumption 1 (the first inclusion property in (4) is verified). Then, consider the time derivative of $V$ :

$$
\begin{aligned}
\dot{V}= & \dot{x}^{\top} P x+x^{\top} P \dot{x}+2 \sum_{j=1}^{M} \dot{x}^{\top} \Lambda^{j} F_{j}(x) \\
= & x^{\top}\left(A_{0}^{\top} P+P A_{0}\right) x+\left(\sum_{j=1}^{M} F_{j}(x)^{\top} A_{j}^{\top}\right) P x+x^{\top} P \sum_{j=1}^{M} A_{j} F_{j}(x)+2 x^{\top} P \varphi \\
& +2 \sum_{j=1}^{M}\left(x^{\top} A_{0}^{\top} \Lambda^{j} F_{j}(x)+\varphi^{\top} \Lambda^{j} F_{j}(x)+\left(\sum_{s=1}^{M} F_{s}(x)^{\top} A_{s}^{\top}\right) \Lambda^{j} F_{j}(x)\right) .
\end{aligned}
$$

Therefore, under (14) we obtain

$$
\begin{aligned}
\dot{V}= & {\left[\begin{array}{c}
x \\
F_{1}(x) \\
\vdots \\
F_{M}(x) \\
\varphi
\end{array}\right]^{\top} Q\left[\begin{array}{c}
x \\
F_{1}(x) \\
\vdots \\
F_{M}(x) \\
\varphi
\end{array}\right]-x^{\top} \Xi^{0} x-\sum_{j=1}^{M} F_{j}(x)^{\top} \Xi^{j} F_{j}(x)-2 \sum_{j=1}^{M} x^{\top} \Upsilon_{0, j} F_{j}(x) } \\
& -2 \sum_{s=1}^{M-1} \sum_{r=s+1}^{M} F_{s}(x)^{\top} \Upsilon_{s, r} F_{r}(x)+\varphi^{\top} \Phi \varphi \\
\leq & -x^{\top} \Xi^{0} x-\sum_{j=1}^{M} F_{j}(x)^{\top} \Xi^{j} F_{j}(x)-2 \sum_{j=1}^{M} x^{\top} \Upsilon_{0, j} F_{j}(x) \\
& -2 \sum_{s=1}^{M-1} \sum_{r=s+1}^{M} F_{s}(x)^{\top} \Upsilon_{s, r} F_{r}(x)+\varphi^{\top} \Phi \varphi,
\end{aligned}
$$

where the right-hand side of the last inequality is radially unbounded as a function of $x$ under the condition (16) and Assumption 11. then the second relation in (4) can be recovered and the ISS property of the system (11) is guaranteed. Therefore, all solutions of the system are bounded.

Next, we need to establish the asymptotic stability of the dynamics $(13)$ that implies existence of a globally attracting solution in $(11)$, for which a Lyapunov function

$$
\tilde{V}(z)=z^{\top} \tilde{\boldsymbol{P}} z+2 \sum_{j=1}^{M} \sum_{i=1}^{n} \tilde{\Lambda}^{j, i} \int_{0}^{z_{i}} f_{j}^{i}(\tau) d \tau
$$


is considered, and its positive definiteness and radial unboundedness in the variable $z$ follows the same argumentation as before, whose time derivative admits the following representation:

$$
\begin{aligned}
\dot{\tilde{V}}= & {\left[\begin{array}{c}
z \\
G_{1}(x, z) \\
\vdots \\
G_{M}(x, z) \\
F_{1}(z) \\
\vdots \\
F_{M}(z)
\end{array}\right]^{\top}\left[\begin{array}{c}
z \\
G_{1}(x, z) \\
\vdots \\
G_{M}(x, z) \\
F_{1}(z) \\
\vdots \\
F_{M}(z)
\end{array}\right]+\gamma \sum_{j=1}^{M} G_{j}(x, z)^{\top} G_{j}(x, z)+\theta \sum_{j=1}^{M} F_{j}^{\top}(z) F_{j}(z) } \\
& -z^{\top} \tilde{\Xi}^{0} z-2 \sum_{j=1}^{M} z^{\top} \Gamma_{j} G_{j}(x, z)-2 \sum_{j=1}^{M} z^{\top} \Omega_{j} F_{j}(z)-2 \sum_{j=1}^{M} \sum_{j^{\prime}=1}^{M} G_{j}(x, z)^{\top} \tilde{\Upsilon}_{j, j^{\prime}} F_{j^{\prime}}(z) .
\end{aligned}
$$

Since $\tilde{Q} \leq 0$ and applying Assumption 3 , we get

$$
\begin{aligned}
\dot{\tilde{V}} \leq & -z^{\top}\left(\tilde{\Xi}^{0}-\gamma \sum_{j=1}^{M} S_{0}^{j}-\theta \sum_{j=1}^{M} H_{0}^{j}\right) z-2 \sum_{j=1}^{M} z^{\top}\left(\Gamma_{j}-\gamma S_{1}^{j}-\theta H_{1}^{j}\right) G_{j}(x, z) \\
& -2 \sum_{j=1}^{M} z^{\top}\left(\Omega_{j}-\gamma S_{2}^{j}-\theta H_{2}^{j}\right) F_{j}(z)-2 \sum_{j=1}^{M} G_{j}(x, z)^{\top} \sum_{j^{\prime}=1}^{M}\left(\tilde{\Upsilon}_{j, j^{\prime}}-\gamma S_{3}^{j, j^{\prime}}-\theta H_{3}^{j, j^{\prime}}\right) F_{j^{\prime}}(z) .
\end{aligned}
$$

The second and the fourth terms in the above estimate are nonnegative due to Assumption 2. The SIIOS characteristics in (6) are verified due to (17). Therefore, we can substantiate that the system (11), (13) is SIIOS with respect to $z$, then there exists $\beta \in \mathscr{K} \mathscr{L}$ such that

$$
\left\|z\left(t, z_{0}, 0\right)\right\| \leq \beta\left(\left\|z_{0}\right\|, t\right), \forall t \in \mathbb{R}_{+}
$$

for any $z_{0} \in \mathbb{R}^{n}$. Finally, (11) has all solutions bounded and converging to a unique globally attracting one, which describes the convergence property due to Definition 7 .

Remark 2. Note that Assumption 3 is used only in the second part of the proof of Theorem 4, where the ISS property of the system (11) has been already established. It means that despite the assumption is formulated for all $x, y \in \mathbb{R}^{n}$, the variable $x$ (that represents the limit motion) can be assumed to be bounded, which enlarges greatly the class of admissible nonlinearities verifying Assumption 3 . The current formulation of the assumption is chosen for brevity of exposition.

Remark 3. To prove Theorem 4 in the first part, it is enough to substantiate global stability of trajectories for the system (11), then conditions of ISS can be relaxed. For example, all bounded nonlinearities can be treated as a part of the input $\varphi$, or some cross-terms including such nonlinearities, as $\varphi^{\top} \Lambda^{j} F_{j}(x)$ for instance, can be hidden in $\varphi$, which may sparse the matrix $Q$.

Remark 4. It is also possible to use IOS/ROS properties to show the convergence for the system (13) (see Theorem 2 and Remark 1 for proof). Let

$$
H(z)=z
$$

be the output of (13) and consider IOS or ROS property of the system (11), (13), (19). We see that the system (11), (13), (19) is with zero input, the conditions (7), (9) and (10) can be verified. Thus, all solutions of (13) are bounded by

$$
\left\|z\left(t, z_{0}, 0\right)\right\| \leq \beta\left(\left\|z_{0}\right\|, t\right)
$$

for some $\beta \in \mathscr{K} \mathscr{L}$, all $z_{0} \in \mathbb{R}^{n}$ and $t \geq 0$.

Corollary 1. Let $\varphi \in \mathscr{L}_{\infty}^{n}$ be an almost periodic function for $t \in \mathbb{R}$, the functions $F_{j}, j \in \overline{1, M}$ be locally Lipschitz continuous and all conditions of Theorem 4 be verified, then the system (11) possesses almost periodic convergence property.

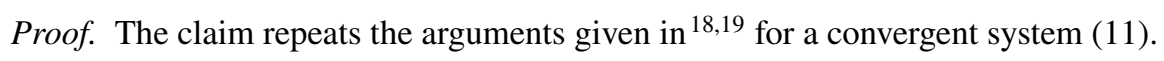

\section{2 | Case of Metzler matrices}

In this subsection, we consider convergence and almost periodic convergence conditions for the system (11) with coefficient matrices satisfying the following assumption: 
Assumption 4. Assume that the matrices $A_{s} \in \mathbb{R}^{n \times n}, s \in \overline{0, M}$ are Metzler and there exists a vector $v>0$ in $\mathbb{R}^{n}$ with $v^{\top} A_{s}=-c_{s}^{\top} \leq 0$ for $s \in \overline{0, M}$ and $\sum_{s^{\prime}=0}^{m} c_{s^{\prime}}>0$.

Note that we do not require that all matrices $A_{s}$ with $s \in \overline{0, M}$ be Hurwitz, but only its total composition, evaluated by $\sum_{s^{\prime}=0}^{m} c_{s^{\prime}}$, has to provide the stability. The proofs of the results of this subsection can be found in $\frac{35}{\text {. }}$

Theorem 5. If assumptions 1,2 and 4 are fulfilled, then the system (11) is convergent.

In this case, Assumption 4 replaces all LMIs given in the formulation of Theorem 4

Corollary 2. Let $\varphi \in \mathscr{L}_{\infty}^{n}$ be an almost periodic function for $t \in \mathbb{R}$, the functions $F_{j}, j \in \overline{1, M}$ be locally Lipschitz continuous and assumptions 1, 2, and 4 be fulfilled, then the system (11) possesses almost periodic convergence property.

\section{5 | CONVERGENCE CONDITIONS FOR DISCRETIZED SYSTEMS}

In this section, the convergence conditions for two kinds of discretized systems are given, which are obtained from application of two discretization approaches to the system (11), namely the explicit and the implicit Euler methods are studied. Only the convergence property is investigated, the almost periodic one can be obtained by adapting the conditions of $\frac{36}{36}$ and corollaries 1 or 2 (it is skipped for brevity).

Assumption 5. Assume that for every $i \in \overline{1, n}, j \in \overline{1, M}$, the function $f_{j}^{i}$ is globally Lipschitz continuous, i.e., there exists a number $L_{j}^{i}>0$ such that

$$
\left|f_{j}^{i}\left(r_{1}\right)-f_{j}^{i}\left(r_{2}\right)\right| \leq L_{j}^{i}\left|r_{1}-r_{2}\right|
$$

for all $r_{1}, r_{2} \in \mathbb{R}$.

\section{1 | Explicit Euler method}

Consider the difference system

$$
x(k+1)=x(k)+h A_{0} x(k)+h \sum_{j=1}^{M} A_{j} F_{j}(x(k))+h \varphi(k),
$$

where $h>0$ is a discretization step; $k_{0} \in \mathbb{Z}$ and $k_{0} \leq k \in \mathbb{Z} ; \varphi \in \mathscr{U}_{\infty}^{n} ; x(k)$ and $F_{j}, j \in \overline{1, M}$ are defined as in the system (11).

It is clear that system (20) is the result of discretization of (11) with the aid of the explicit Euler method.

Theorem 6. Let assumptions 1, 2, 3 and 5 be satisfied. If there exist $0 \leq P=P^{\top}, \tilde{P}=\tilde{P}^{\top} \in \mathbb{R}^{n \times n} ;\left\{\Lambda^{j}=\right.$ $\left.\operatorname{diag}\left(\Lambda^{j, 1}, \ldots, \Lambda^{j, n}\right)\right\}_{j=1}^{M}, \quad\left\{\tilde{\Lambda}^{j}=\operatorname{diag}\left(\Lambda^{j, 1}, \ldots, \tilde{\Lambda}^{j, n}\right)\right\}_{j=1}^{M}, \quad\left\{\Xi^{s}\right\}_{s=0}^{M}, \quad\left\{\Upsilon_{s, r}\right\}_{0 \leq s<r \leq M},\left\{\tilde{\Upsilon}_{j, j^{\prime}}\right\}_{j, j^{\prime}=1}^{M}, \quad\left\{\Gamma_{j}\right\}_{j=1}^{M}, \quad\left\{\Omega_{j}\right\}_{j=1}^{M} \subset \mathbb{D}_{+}^{n} ;$ $\tilde{\Xi}^{0} \in \mathbb{D}_{+}^{n} ; 0<\Phi=\Phi^{\top} \in \mathbb{R}^{n \times n}, \gamma, \theta>0$ such that the properties (14)-(17) are verified for

$$
Q=\left(Q_{a, b}\right)_{a, b=1}^{M+2}, \tilde{Q}=\left(\tilde{Q}_{a, b}\right)_{a, b=1}^{3}
$$

with

$$
\begin{gathered}
Q_{1,1}=A_{0}^{\top} P+P A_{0}+h A_{0}^{\top} \Pi A_{0}+\Xi^{0}, \\
Q_{j+1, j+1}=h A_{j}^{\top} \Pi A_{j}+\Lambda^{j} A_{j}+A_{j}^{\top} \Lambda^{j}+\Xi^{j}, j \in \overline{1, M}, \\
Q_{1, j+1}=P A_{j}+A_{0}^{\top} \Lambda^{j}+h A_{0}^{\top} \Pi A_{j}+\Upsilon_{0, j}, j \in \overline{1, M}, \\
Q_{s+1, r+1}=h A_{s}^{\top} \Pi A_{r}+A_{s}^{\top} \Lambda^{r}+\Lambda^{s} A_{r}+\Upsilon_{s, r}, s \in \overline{1, M-1}, r \in \overline{s+1, M}, \\
Q_{M+2, M+2}=h \Pi-\Phi ; Q_{j+1, M+2}=h A_{j}^{\top} \Pi+\Lambda^{j}, j \in \overline{1, M}, \\
Q_{1, M+2}=P+h A_{0}^{\top} \Pi, \Pi=P+2 \sum_{j=1}^{M} L_{j} \Lambda^{j}, L_{j}:=\operatorname{diag}\left(L_{j}^{1}, \ldots, L_{j}^{n}\right), j \in \overline{1, M},
\end{gathered}
$$




$$
\begin{gathered}
\tilde{Q}_{1,1}=A_{0}^{\top} P+P A_{0}+h A_{0}^{\top} \tilde{\Pi} A_{0}+\tilde{\Xi}^{0}, \\
\tilde{Q}_{2,2}=h A^{\top} \tilde{\Pi} A-\gamma I_{n M}, \tilde{Q}_{1,2}=h A_{0}^{\top} \tilde{\Pi} A+P A+\Gamma, \\
\tilde{Q}_{1,3}=A_{0}^{\top} \tilde{\Lambda}+\Omega, \tilde{Q}_{2,3}=A^{\top} \tilde{\Lambda}+\tilde{\Upsilon}, \tilde{Q}_{3,3}=-\theta I_{n M}, \\
\tilde{\Pi}=P+2 \sum_{j=1}^{M} L_{j} \tilde{\Lambda}^{j}, A=\left[\begin{array}{lll}
A_{1} & \ldots & A_{M}
\end{array}\right], \Gamma=\left[\begin{array}{lll}
\Gamma_{1} & \ldots & \Gamma_{M}
\end{array}\right], \\
\tilde{\Lambda}=\left[\begin{array}{llll}
\tilde{\Lambda}^{1} & \ldots & \tilde{\Lambda}^{M}
\end{array}\right], \Omega=\left[\begin{array}{lll}
\Omega_{1} & \ldots & \Omega_{M}
\end{array}\right], \tilde{\Upsilon}=\left(\tilde{\Upsilon}_{j, j^{\prime}}\right)_{j, j^{\prime}=1}^{M},
\end{gathered}
$$

then the system 20 is convergent.

Proof. Consider a candidate Lyapunov function

$$
V(x(k))=x(k)^{\top} P x(k)+2 \sum_{j=1}^{M} \sum_{i=1}^{n} \Lambda^{j, i} \int_{0}^{x_{i}(k)} f_{j}^{i}(\tau) d \tau
$$

for the system (20), so the first condition in $(8)$ is verified as before. Then, consider the difference of $V$ :

$$
\begin{aligned}
& \Delta V:=x(k+1)^{\top} \boldsymbol{P} x(k+1)-x(k)^{\top} \boldsymbol{P} x(k)+2 \sum_{j=1}^{M} \sum_{i=1}^{n} \Lambda^{j, i} \int_{x_{i}(k)}^{x_{i}(k+1)} f_{j}^{i}(\tau) d \tau \\
& =\left[x(k+1)^{\top} \boldsymbol{P} x(k+1)-x(k)^{\top} \boldsymbol{P} x(k)\right]+2 h\left[\sum_{j=1}^{M} F_{j}(x(k))^{\top} \Lambda^{j} T(k)\right] \\
& +2\left[\sum_{j=1}^{M} G_{j}(x(k), \varsigma(k)-x(k))^{\top} \Lambda^{j}(x(k+1)-x(k))\right] \\
& \leq\left[x(k+1)^{\top} \operatorname{Px}(k+1)-x(k)^{\top} \boldsymbol{P} x(k)\right]+2 h\left[\sum_{j=1}^{M} F_{j}(x(k))^{\top} \Lambda^{j} T(k)\right] \\
& +2 h^{2}\left[T(k)^{\top}\left(\sum_{j=1}^{M} L_{j} \Lambda^{j}\right) T(k)\right] \\
& =h\left[\begin{array}{c}
x(k) \\
F_{1}(x(k)) \\
\vdots \\
F_{M}(x(k)) \\
\varphi(k)
\end{array}\right]^{\top} Q\left[\begin{array}{c}
x(k) \\
F_{1}(x(k)) \\
\vdots \\
F_{M}(x(k)) \\
\varphi(k)
\end{array}\right]-h x(k)^{\top} \Xi^{0} x(k) \\
& -h \sum_{j=1}^{M} F_{j}(x(k))^{\top} \Xi^{j} F_{j}(x(k))-2 h \sum_{j=1}^{M} x(k)^{\top} \Upsilon_{0, j} F_{j}(x(k)) \\
& -2 h \sum_{s=1}^{M-1} \sum_{r=s+1}^{M} F_{s}(x(k))^{\top} \Upsilon_{s, r} F_{r}(x(k))+h \varphi(k)^{\top} \Phi \varphi(k)
\end{aligned}
$$

by Assumption 5 and the Mean Value Theorem, where $T(k)=A_{0} x(k)+\sum_{j=1}^{M} A_{j} F_{j}(x(k))+\varphi(k)$ and $\varsigma_{i}(k) \in\left[\min \left(x_{i}(k), x_{i}(k+\right.\right.$ $\left.1)), \max \left(x_{i}(k), x_{i}(k+1)\right)\right], i \in \overline{1, n}$ for all $k \geq k_{0}$. Therefore, with the condition (14) we obtain

$$
\begin{aligned}
\Delta V \leq & -h x^{\top} \Xi^{0} x-h \sum_{j=1}^{M} F_{j}(x)^{\top} \Xi^{j} F_{j}(x)-2 h \sum_{j=1}^{M} x^{\top} \Upsilon_{0, j} F_{j}(x) \\
& -2 h \sum_{s=1}^{M-1} \sum_{r=s+1}^{M} F_{s}(x)^{\top} \Upsilon_{s, r} F_{r}(x)+h \varphi^{\top} \Phi \varphi,
\end{aligned}
$$

so that under assumptions 1,2 and the condition (16) the system (20) is ISS. 
In a similar way, we then study the asymptotic stability of

$$
z(k+1)=z(k)+h A_{0} z(k)+h \sum_{j=1}^{M} A_{j} G_{j}(x(k), z(k))
$$

with $y(k+1)=y(k)+h A_{0} y(k)+h \sum_{j=1}^{M} A_{j} F_{j}(y(k))+h \varphi(k)$ and $z(k)=y(k)-x(k)$ for all $k \geq k_{0}$, for which we consider a Lyapunov function

$$
\tilde{V}(z(k))=z(k)^{\top} \tilde{P} z(k)+2 \sum_{j=1}^{M} \sum_{i=1}^{n} \tilde{\Lambda}^{j, i} \int_{0}^{z_{i}(k)} f_{j}^{i}(\tau) d \tau
$$

for the dynamics (21). Under the repetition of previous development in the proof for Theorem 4 it follows that under assumptions 1.,2, 3, 5] and the conditions (15), (17)

$$
\begin{aligned}
\Delta \tilde{V} \leq & -h z^{\top}\left(\tilde{\Xi}^{0}-\gamma \sum_{j=1}^{M} S_{0}^{j}-\theta \sum_{j=1}^{M} H_{0}^{j}\right) z-2 h \sum_{j=1}^{M} z^{\top}\left(\Gamma_{j}-\gamma S_{1}^{j}-\theta H_{1}^{j}\right) G_{j}(x, z)-2 h \sum_{j=1}^{M} z^{\top}\left(\Omega_{j}-\gamma S_{2}^{j}-\theta H_{2}^{j}\right) F_{j}(z) \\
& -2 h \sum_{j=1}^{M} G_{j}(x, z)^{\top} \sum_{j^{\prime}=1}^{M}\left(\tilde{\Upsilon}_{j, j^{\prime}}-\gamma S_{3}^{j, j^{\prime}}-\theta H_{3}^{j, j^{\prime}}\right) F_{j^{\prime}}(z) \\
< & 0
\end{aligned}
$$

for all $z \neq 0$ and $x \in \mathbb{R}^{n}$. Therefore, 21] is globally asymptotically stable (GAS). This completes the proof.

Note that if Assumption 5 holds only locally, i.e., the nonlinear functions in 201 are locally Lipschitz, then local convergence can be established in the same way.

The result of Theorem 6 can be reformulated as follows:

Corollary 3. Let all conditions of Theorem 4 be satisfied with Assumption 5 Then there exists $h_{0}>0$ such that for all $h \in\left(0, h_{0}\right]$ the system $[20)$ is convergent.

Proof. It is easy to check that the difference between matrices $Q$ and $\tilde{Q}$ given in theorems 4 and 6 is proportional to $h$, and all other conditions are the same, except Assumption 5 that is additionally imposed in the last theorem. This substantiates the claim.

\section{2 | Implicit Euler method}

In this subsection the convergence conditions for a discretization of (11) with the aid of implicit Euler method is investigated. The considered discretized system is

$$
x(k+1)=x(k)+h A_{0} x(k+1)+h \sum_{j=1}^{M} A_{j} F_{j}(x(k+1))+h \varphi(k+1),
$$

where $x(k), h, k, F_{j}, j \in \overline{1, M}$ and $\varphi(k+1)$ are defined as in the system [20], and it has to be resolved with respect to $x(k+1)$. Remark 5. In the sequel we assume that for any $h>0$ and $\zeta, \varphi \in \mathbb{R}^{n}$, the equation

$$
\left(I_{n}-h A_{0}\right) \xi-h \sum_{j=1}^{M} A_{j} F_{j}(\xi)=\zeta+h \varphi
$$

admits a unique solution $\xi=\xi(h, \zeta, \varphi)$ that continuously depends on $h, \zeta, \varphi$. Hence, for any $k_{0} \in \mathbb{Z}$ and $x\left(k_{0}\right)$, the system 22) admits a unique solution $x\left(k, x\left(k_{0}\right), k_{0}, \varphi\right)$ that is defined for all $k_{0} \leq k \in \mathbb{Z}$. Sufficient conditions of such a property (under assumptions 1 and 2 with $M=1$ ) were obtained in 3738 .

Theorem 7. Let assumptions 1 and 2 be satisfied. If there exist $0<P=P^{\top} \in \mathbb{R}^{n \times n} ;\left\{\Xi^{s}\right\}_{s=0}^{M},\left\{\Upsilon_{s, r}\right\}_{0 \leq s<r \leq M} \subset \mathbb{D}_{+}^{n}$ and $0<\Phi=\Phi^{\top} \in \mathbb{R}^{n \times n}$ such that

$$
\begin{gathered}
Q=Q^{\top} \leq 0 \\
\Xi^{0}+\sum_{j=1}^{M} \Upsilon_{0, j}+\sum_{s=1}^{m} \Xi^{s}+\sum_{s=1}^{m} \sum_{r=s+1}^{m} \Upsilon_{s, r}>0
\end{gathered}
$$


where

$$
Q=\left(Q_{a, b}\right)_{a, b=1}^{M+2}
$$

with

$$
\begin{gathered}
Q_{1,1}=A_{0}^{\top} P+P A_{0}-h A_{0}^{\top} P A_{0}+\Xi^{0}, \\
Q_{j+1, j+1}=-h A_{j}^{\top} P A_{j}+\Xi^{j}, j \in \overline{1, M}, \\
Q_{1, j+1}=P A_{j}-h A_{0}^{\top} P A_{j}+\Upsilon_{0, j}, j \in \overline{1, M}, \\
Q_{s+1, r+1}=-h A_{s}^{\top} P A_{r}+\Upsilon_{s, r}, s \in \overline{1, M-1}, r \in \overline{s+1, M}, \\
Q_{1, M+2}=P-h A_{0}^{\top} P, \\
Q_{M+2, M+2}=-h P-\Phi ; Q_{j+1, M+2}=-h A_{j}^{\top} P, j \in \overline{1, M},
\end{gathered}
$$

then the system 22) is convergent.

Proof. Consider a candidate Lyapunov function

$$
V(x(k))=x(k)^{\top} P x(k)
$$

for (22), again the first condition in (8) is recovered. Then, the increment of such a $V$ for (22) takes the form:

$$
\begin{aligned}
\Delta V= & x(k+1)^{\top} P x(k+1)-x(k)^{\top} P x(k) \\
= & h\left[\begin{array}{c}
x(k+1) \\
F_{1}(x(k+1)) \\
\vdots \\
F_{M}(x(k+1)) \\
\varphi(k+1)
\end{array}\right]^{\top}\left[\begin{array}{c}
x(k+1) \\
F_{1}(x(k+1)) \\
\vdots \\
F_{M}(x(k+1)) \\
\varphi(k+1)
\end{array}\right]-h x(k+1)^{\top} \Xi^{0} x(k+1) \\
& -h \sum_{j=1}^{M} F_{j}(x(k+1))^{\top} \Xi^{j} F_{j}(x(k+1))-2 h \sum_{s=1}^{M-1} \sum_{r=s+1}^{M} F_{s}(x(k+1))^{\top} \Upsilon_{s, r} F_{r}(x(k+1)) \\
& -2 h \sum_{j=1}^{M} x(k+1)^{\top} \Upsilon_{0, j} F_{j}(x(k+1))+h \varphi(k+1)^{\top} \Phi \varphi(k+1) .
\end{aligned}
$$

Therefore, under 24] we have

$$
\begin{aligned}
\Delta V \leq & -h x(k+1)^{\top} \Xi^{0} x(k+1)-h \sum_{j=1}^{M} F_{j}(x(k+1))^{\top} \Xi^{j} F_{j}(x(k+1)) \\
& -2 h \sum_{s=1}^{M-1} \sum_{r=s+1}^{M} F_{s}(x(k+1))^{\top} \Upsilon_{s, r} F_{r}(x(k+1))-2 h \sum_{j=1}^{M} x(k+1)^{\top} \Upsilon_{0, j} F_{j}(x(k+1)) \\
& +h \varphi(k+1)^{\top} \Phi \varphi(k+1),
\end{aligned}
$$

hence, with the condition (25) the system 22) is ISS (recall Remark5).

Similarly, for the dynamics

$$
z(k+1)=z(k)+h A_{0} z(k+1)+h \sum_{j=1}^{M} A_{j} G_{j}(x(k+1), z(k+1))
$$


with $y(k+1)=y(k)+h A_{0} y(k+1)+h \sum_{j=1}^{M} A_{j} F_{j}(y(k+1))+h \varphi(k+1)$ and $z(k)=y(k)-x(k)$ for all $k \geq k_{0}$, by considering $\tilde{V}(z)=z^{\top} \tilde{P} z$ and following the previous proof lines we obtain

$$
\begin{aligned}
\Delta \tilde{V} \leq & -h z(k+1)^{\top} \Xi^{0} z(k+1)-h \sum_{j=1}^{M} G_{j}(x(k+1), z(k+1))^{\top} \Xi^{j} G_{j}(x(k+1), z(k+1)) \\
& -2 h \sum_{j=1}^{M} z(k+1)^{\top} \Upsilon_{0, j} G_{j}(x(k+1), z(k+1)) \\
& -2 h \sum_{s=1}^{M-1} \sum_{r=s+1}^{M} G_{s}(x(k+1), z(k+1))^{\top} \Upsilon_{s, r} G_{r}(x(k+1), z(k+1)) \\
< & 0
\end{aligned}
$$

for all $z(k+1) \neq 0$ and $x(k+1) \in \mathbb{R}^{n}$. Thus, the dynamics $[26$ is GAS. Therefore, we conclude that the system $[22]$ is convergent.

\section{6 | EXAMPLES}

\section{1 | Application to a modified Lotka-Volterra model}

In this subsection, a modified Lotka-Volterra (LV) dynamics is considered, which has been widely investigated in infectious disease, biology, finance, to mention a few subjects ${ }^{39}$. However, the earliest basic model does not reflect some important phenomena, e.g., stable coexistence and predator preference. Therefore, there are many modified LV models, among them the following one considers additionally the mutualistic interactions in different species ${ }^{400}$.

$$
\dot{x}(t)=\operatorname{diag}(x(t))\left(r_{0}+r(t)+A x(t)+A_{2} \phi(x(t))\right), t \in \mathbb{R},
$$

where $x(t) \in \mathbb{R}_{+}^{n}$ is the population sizes of $n$ species; $r_{0} \in \mathbb{R}^{n}$ denotes the birth or mortality rates of the species and $r(t) \in \mathbb{R}^{n}$ is introduced to model the deviations of the rates from the nominal quantities $r_{0} ; A \in \mathbb{R}^{n \times n}$ represents the community matrix and $A_{2} \in \mathbb{R}^{n \times n}$ refers to the net effect of the mutualism ( $A$ and $A_{2}$ are Metzler); the function $\phi: \mathbb{R}_{+}^{n} \rightarrow \mathbb{R}_{+}^{n}$ is diagonal, where $\phi_{i}\left(x_{i}\right)=\frac{x_{i}}{b_{i}+x_{i}}$ with constants $b_{i}>0$ for $i \in \overline{1, n}$.

Assuming existence of a unique equilibrium point $x_{e} \in \mathbb{R}_{+}^{n} \backslash\{0\}$ for $(27)$ with $r(t)=0$, and defining

$$
\rho(t)=\ln (x(t))-\ln \left(x_{e}\right),
$$

we obtain

$$
\dot{\rho}(t)=A_{1} F_{1}(\rho(t))+A_{2} F_{2}(\rho(t))+r(t),
$$

where

$$
A_{1}:=A \operatorname{diag}\left(x_{e}\right), F_{1}(\rho)=e^{\rho}-\mathbf{1}_{n}, F_{2}(\rho)=\phi\left(\operatorname{diag}\left(x_{e}\right) e^{\rho}\right)-\phi\left(x_{e}\right) .
$$

Note that for such functions $F_{1}$ and $F_{2}$ the requirement of Assumption 1 for $v \rightarrow-\infty$ is not satisfied. However, as in ${ }^{23}$, due to assumed existence of the global equilibrium $x_{e}$ it is possible to show that for $r_{0}+r(t) \geq r_{\min }>0$ all trajectories converge to a neighborhood of the steady state, where the analysis can be next performed without taking into account the unbounded deviations of the state.

For illustration, let

$$
A=\left[\begin{array}{cc}
-0.8 & 0.3 \\
0.4 & -0.6
\end{array}\right], A_{2}=\left[\begin{array}{cc}
-0.5 & 0.2 \\
0.3 & -0.9
\end{array}\right], b=\left[\begin{array}{l}
0.1 \\
0.4
\end{array}\right], r_{0}=\left[\begin{array}{l}
0.2 \\
0.9
\end{array}\right], r(t)=\left[\begin{array}{l}
\sin (t) \\
\cos (t)
\end{array}\right],
$$


where $r(t)$ is periodic and represents the external input for (28), then the value of $x_{e}$ can be obtained as previously mentioned. Assumptions 3 and 5 are satisfied for

$$
\begin{gathered}
S_{0}^{1}=S_{0}^{2}=\left[\begin{array}{cc}
0.0041 & 0 \\
0 & 0.0070
\end{array}\right], S_{1}^{1}=\left[\begin{array}{cc}
0.1177 & 0 \\
0 & 0.1617
\end{array}\right], S_{1}^{2}=\left[\begin{array}{cc}
0.2171 & 0 \\
0 & 0.2907
\end{array}\right], \\
S_{2}^{1}=S_{2}^{2}=10^{-5} \times\left[\begin{array}{cc}
0.0109 & 0 \\
0 & 0.0117
\end{array}\right], S_{3}^{1,1}=S_{3}^{1,2}=\left[\begin{array}{cc}
0.1092 & 0 \\
0 & 0.2105
\end{array}\right], S_{3}^{2,1}=S_{3}^{2,2}=\left[\begin{array}{cc}
0.1545 & 0 \\
0 & 0.3244
\end{array}\right], \\
h_{1}=0.386, h_{2}=0.557, L_{1}=\left[\begin{array}{cc}
40 & 0 \\
0 & 40
\end{array}\right], L_{2}=\left[\begin{array}{cc}
0.0588 & 0 \\
0 & 0.3694
\end{array}\right] .
\end{gathered}
$$

The LMIs proposed in Theorem 4 are verified by

$$
\begin{aligned}
& P=\left[\begin{array}{ll}
0.8054 & 0.2870 \\
0.2870 & 0.7936
\end{array}\right], \tilde{P}=10^{-5} \times\left[\begin{array}{ll}
0.1751 & 0.0762 \\
0.0762 & 0.1153
\end{array}\right], \\
& \Lambda^{1}=10^{8} \times\left[\begin{array}{cc}
0.9572 & 0 \\
0 & 1.7737
\end{array}\right], \Lambda^{2}=10^{8} \times\left[\begin{array}{cc}
1.5435 & 0 \\
0 & 2.4658
\end{array}\right], \tilde{\Lambda}^{1}=\tilde{\Lambda}^{2}=10^{-5} \times\left[\begin{array}{cc}
0.1057 & 0 \\
0 & 0.1144
\end{array}\right],
\end{aligned}
$$

and the ones in theorems 6 and 7 under the selection $h=10^{-4}$ are checked for

$$
\begin{aligned}
& P=\left[\begin{array}{ll}
0.9939 & 0.3007 \\
0.3007 & 0.9362
\end{array}\right], \tilde{P}=10^{-4} \times\left[\begin{array}{ll}
0.1840 & 0.0802 \\
0.0802 & 0.1072
\end{array}\right], \\
& \Lambda^{1}=10^{8} \times\left[\begin{array}{cc}
0.9949 & 0 \\
0 & 1.8803
\end{array}\right], \Lambda^{2}=10^{8} \times\left[\begin{array}{cc}
1.6589 & 0 \\
0 & 2.6251
\end{array}\right] \text {, } \\
& \tilde{\Lambda}^{1}=10^{-4} \times\left[\begin{array}{cc}
0.5388 & 0 \\
0 & 0.6215
\end{array}\right], \tilde{\Lambda}^{2}=10^{-4} \times\left[\begin{array}{cc}
0.5388 & 0 \\
0 & 0.6217
\end{array}\right],
\end{aligned}
$$

and $P=10^{-7} \times\left[\begin{array}{ll}0.2932 & 0.0983 \\ 0.0983 & 0.1739\end{array}\right]$, respectively.

By choosing a periodic and oscillating input signal, we can see a convergence phenomenon that is not caused by asymptotic stability of the considered system. The trajectories with distinct initial states

$$
x^{1}(0)=\left[\begin{array}{l}
2.4 \\
0.7
\end{array}\right], x^{2}(0)=\left[\begin{array}{l}
5.3 \\
0.8
\end{array}\right], x^{3}(0)=\left[\begin{array}{l}
0.1 \\
3.7
\end{array}\right]
$$

are shown in Fig. 1 These results demonstrate the convergence phenomenon in 28.

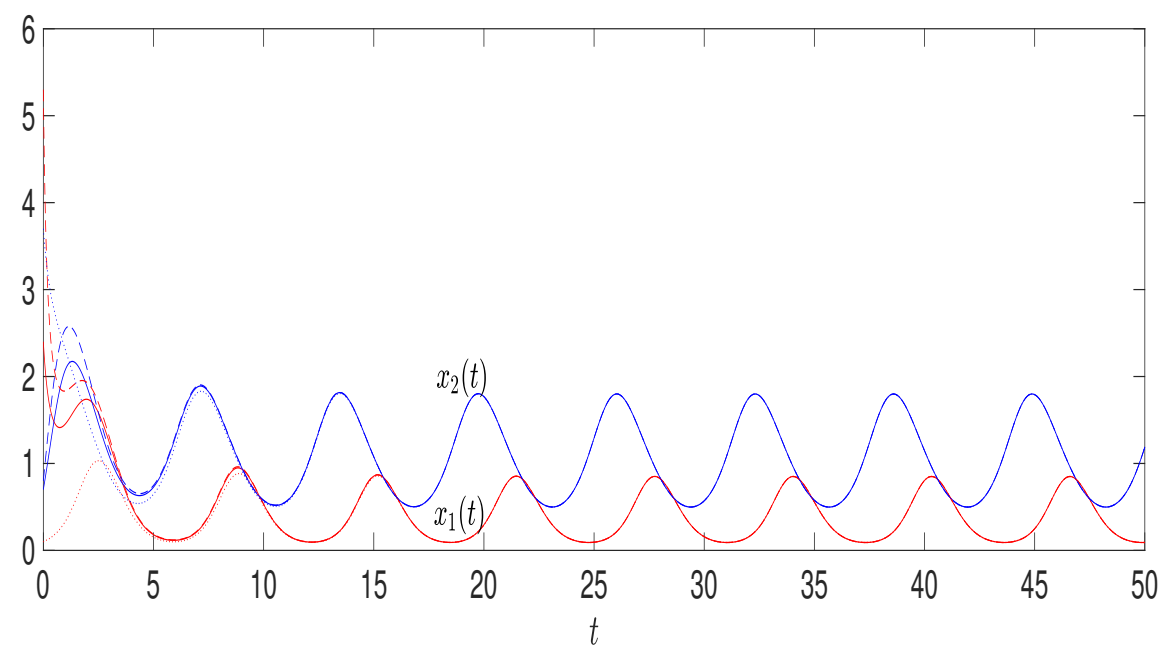

FIGURE 1 The state trajectories of 27] versus the time $t$ 


\section{2 | Application to opinion dynamics}

For opinion dynamics among a network of agents, the following model can be considered ${ }^{41 / 42 / 43}$.

$$
\dot{x}(t)=-x(t)+\sum_{j=1}^{M} K_{j} A_{j} \tanh \left(\alpha_{j} x(t)\right)+B u(t)+\varphi(t),
$$

where $x(t) \in \mathbb{R}^{n}$ is the opinion variable of $n$ agents, and the sign of $x_{i}(t), i=1, \ldots, n$ describes the qualitative stance toward a binary choice (the larger $\left|x_{i}(t)\right|$, more extreme is the opinion of the agent $i$ ); $M \geq 1$ is the number of social networks connecting the agents; $K_{j}>0$ denotes the social interaction strength among agents in the network $j \in \overline{1, M}, A_{j} \in \mathbb{R}^{n \times n}$ is the adjacency matrix in that network, and $\alpha_{j}>0$ characterizes controversialness of the issue for $j^{\text {th }}$ media (for small $\alpha_{j}$, the social influence of moderate individuals on others is weak, while for a large $\alpha_{j}$, everybody have a strong social impact); $B \in \mathbb{R}^{n \times q} ; u(t) \in \mathbb{R}^{q}$ is a controlling input that can be applied to modify the network connections among the agents; $\varphi(t) \in \mathbb{R}^{n}$ can be used to model the off-network influences on orientations of agents (e.g., governmental communication). The detailed motivation for this model (for the case $M=1$ and time-varying matrix $A_{1}$ ) can be found in ${ }^{42433}$. Clearly, the system under feedback control is presented in the form of 11, and assumptions 1, 2, 3 and 5 are verified. If Assumption 4 is also valid, then in the resulting closed-loop system, the opinion of an agent can be changed by the external manipulation $\varphi$ only.

Since all nonlinearities are bounded and $A_{0}=-I_{n}$, the system always has bounded solutions for bounded inputs. Hence, the conditions of Theorem 4 can be simplified, as mentioned in Remark 3, by focusing only on the asymptotic stability of (13).

Let $n=4, M=2$,

$$
\begin{gathered}
A_{1}=\left[\begin{array}{llll}
0 & 1 & 1 & 1 \\
1 & 0 & 0 & 0 \\
1 & 0 & 0 & 0 \\
0 & 0 & 1 & 0
\end{array}\right], A_{2}=\left[\begin{array}{llll}
0 & 0 & 0 & 0 \\
0 & 0 & 1 & 0 \\
0 & 1 & 0 & 1 \\
1 & 0 & 0 & 0
\end{array}\right], B=\left[\begin{array}{cccc}
2 & 1 & 1 & 0 \\
-1 & 0.5 & 0 & -0.1 \\
4 & -0.2 & 3 & 1 \\
2 & 0.1 & 4 & 2
\end{array}\right], K_{1}=2, K_{2}=1, \alpha_{1}=0.5, \alpha_{2}=0.1, \varphi(t)=\left[\begin{array}{c}
0.25 \\
0.6 \\
0.3 \\
0.8
\end{array}\right], \\
u(t)=Z_{1} \tanh \left(\alpha_{1} x(t)\right)+Z_{2} \tanh \left(\alpha_{2} x(t)\right), \\
Z_{1}=\left[\begin{array}{cccc}
1.1107 & 1.2711 & -0.3044 & -1.0206 \\
3.1045 & 0.7848 & -0.4515 & -2.8485 \\
-5.4459 & -3.667 & 0.0203 & 3.4097 \\
10.6159 & 6.4136 & -0.4136 & -6.6364
\end{array}\right], Z_{2}=\left[\begin{array}{cccc}
-1.3417 & 0.5825 & 1.0519 & -0.1570 \\
-2.8894 & 1.922 & 2.2015 & 0.3864 \\
4.6529 & -2.2669 & -3.6353 & 0.5077 \\
-8.2295 & 4.0852 & 6.2886 & -0.9977
\end{array}\right] .
\end{gathered}
$$

The trajectories with different initial conditions are presented in Fig. 2 and the LMIs in Theorem 4 are verified. As we can conclude from these results, the opinions of all agents are directed to the predetermined by $\varphi$ direction. Simulations of the same system with $u=0$ and $\varphi=0$ demonstrate pluralism of opinions in the uncontrolled network depending on the initial conditions.

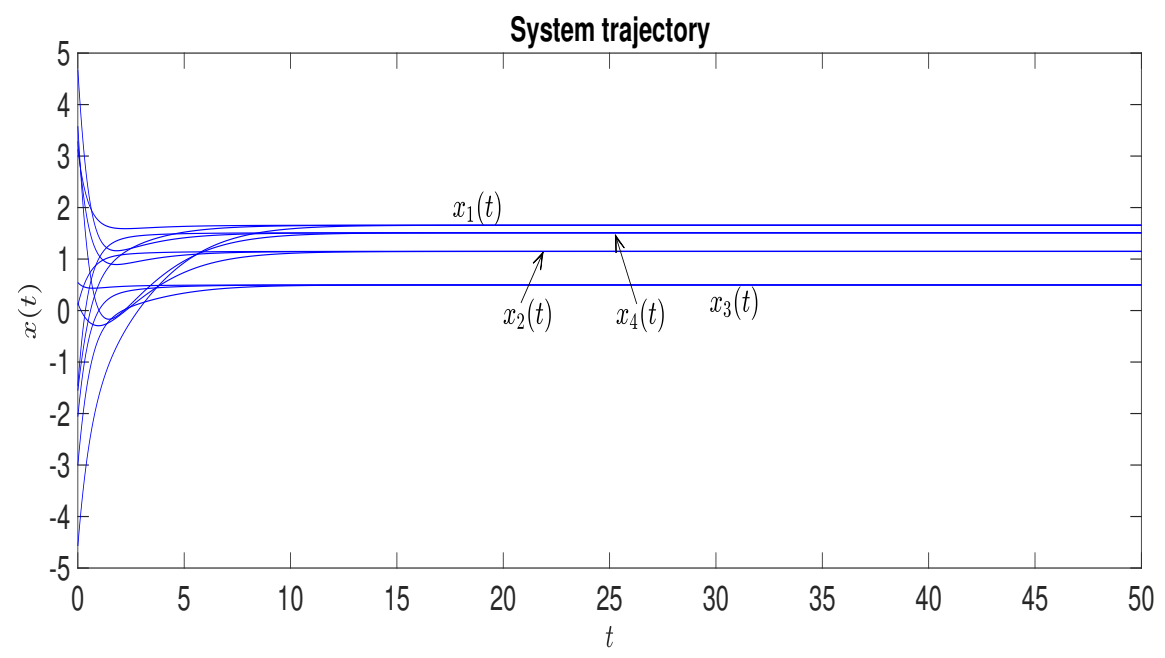

FIGURE 2 The state trajectories of (29) versus the time $t$ 


\section{7 | CONCLUSION}

In this paper, broad convergence conditions for generalized Persidskii systems and their discretizations, and the auxiliary results of convergence and almost periodic convergence conditions for those systems, with Metzler coefficient matrices, were proposed. The formulated conditions were obtained in the form of LMIs (linear inequalities in the Metzler case), which can be constructively verified. The simulations of Lotka-Volterra models and opinion dynamics were presented for the examination of the proposed results.

Future directions of research include the analysis of more sophisticated Lyapunov functions and the investigation of robust convergence properties, and the influence of delays as well.

\section{ACKNOWLEDGMENT}

This work was partially supported by the China Scholarship Council (CSC) Scholarship (CSC No. 201908050104), and by the Ministry of Science and Higher Education of Russian Federation, passport of goszadanie no. 2019-0898.

\section{CONFLICT OF INTEREST}

The authors declare no potential conflict of interests.

\section{DATA AVAILABILITY STATEMENT}

Data sharing not applicable to this article as no datasets were generated or analyzed during the current study.

\section{References}

1. Pavlov A, Wouw N, Nijmeijer H. Convergent Systems: Analysis and Synthesis. In: Berlin/Heidelberg: Springer-Verlag. 2005 (pp. 131-146).

2. Pavlov A, Wouw v. dN. Convergent Systems: Nonlinear Simplicity. In: . 470 of 2017 Lecture Notes in Control and Information Sciences. 2017 (pp. 51-77).

3. Angeli D. A Lyapunov approach to incremental stability properties. IEEE Transactions on Automatic Control 2002; 47 : 410-421.

4. Rüffer BS, Wouw v. dN, Mueller M. Convergent systems vs. incremental stability. Systems \& Control Letters 2013; 62: $277-285$

5. Jouffroy J, Fossen T. Tutorial on Incremental Stability Analysis using Contraction Theory. Modeling, Identification and Control: A Norwegian Research Bulletin 2010; 31: 93-106.

6. Pavlov A, Wouw v. dN, Nijmeijer H. Uniform Output Regulation of Nonlinear Systems. Boston, MA: Birkhäuser Boston . 2006.

7. Pogromsky AY. Passivity Based Design of Synchronizing Systems. International Journal of Bifurcation and Chaos 1998; 08: 295-319.

8. Cai C, Chen G. Synchronization of complex dynamical networks by the incremental ISS approach. Physica A: Statistical Mechanics and its Applications 2006; 371: 754-766.

9. Stan GB, Sepulchre R. Analysis of Interconnected Oscillators by Dissipativity Theory. IEEE Transactions on Automatic Control 2007; 52: 256-270. 
10. Pavlov A, Wouw v. dN, Nijmeijer H. Frequency Response Functions for Nonlinear Convergent Systems. IEEE Transactions on Automatic Control 2007; 52: 1159-1165.

11. Liu RW. Convergent systems. IEEE Transactions on Automatic Control 1968; 13: 384-391.

12. Kosov AA. Investigation of convergence of large scale almost periodic systems by means of comparison vector functions with components as forms of even degrees. Russian Mathematics 2015; 59(7): 21-30.

13. Kosov AA, Shchennikov VN. On the convergence phenomenon in complex almost periodic systems. Differential Equations 2014; 50(12): 1573-1583.

14. Khalil H. Nonlinear systems. Upper Saddle River, NJ: Prentice-Hall . 2002.

15. Levitan B, Zhikov V. Almost periodic funcitons and differential equations. New York: Cambridge University Press . 1982.

16. Pliss VA. Nonlocal problems of the theory of oscillations. London: Academic Press . 1966.

17. Pavlov A, Pogromsky A, Wouw v. dN, Nijmeijer H. Convergent dynamics, a tribute to Boris Pavlovich Demidovich. Systems \& Control Letters 2004; 52: 257-261.

18. Zubov VI. Oscillations in Nonlinear and Controlled Systems. Leningrad: Sudpromgiz . 1962. in Russian.

19. Zubov VI. Theory of Oscillations. World Scientific . 1999.

20. Yoshizawa T. Stability theory by Lyapunov's second method. Mathematical Society of Japan . 1966.

21. Yakubovich VA. Method of matrix unequalities in theory of nonlinear control systems stability. I. Forced oscillations absolute stability. Avtomat. i Telemekh. 1964; 25: 1017-1029.

22. Hallam TG. Convergence of solutions of perturbed nonlinear differential equations. Annali di Matematica Pura ed Applicata 1972; 94: 275-282.

23. Aleksandrov A, Aleksandrova E. Convergence conditions for some classes of nonlinear systems. Systems \& Control Letters 2017; 104: 72-77.

24. Efimov D, Polyakov A, Levant A, Perruquetti W. Realization and Discretization of Asymptotically Stable Homogeneous Systems. IEEE Transactions on Automatic Control 2017; 62(11): 5962-5969.

25. Polyakov A, Efimov D, Brogliato B. Consistent Discretization of Finite-Time and Fixed-Time Stable Systems. SIAM Journal on Control and Optimization 2019; 57(1): 78-103.

26. Barbashin E. On construction of Lyapunov functions for nonlinear systems. Proc. 1st IFAC World Congress 1961: $742-751$.

27. Persidskii S. Concerning problem of absolute stability. Automation and Remote Control 1969: 5-11.

28. Efimov D, Aleksandrov A. Robust stability analysis and implementation of Persidskii systems. 58th IEEE Conference on Decision and Control (CDC) 2019.

29. Mei W, Efimov D, Ushirobira R. Feedback synchronization in Persidskii systems. Proc. 21st IFAC World Congress 2020.

30. Kaszkurewicz E, Bhaya A. Matrix Diagonal Stability in Systems and Computation. Boston, MA: Birkhäuser Boston . 2000.

31. Sontag E, Wang Y. Lyapunov Characterizations of Input to Output Stability. SIAM Journal on Control and Optimization 2000; 39(1): 226-249.

32. Jiang ZP, Sontag E, Wang Y. Input-to-state stability for discrete-time nonlinear systems. IFAC Proceedings Volumes 1999; 32: $2403-2408$.

33. Sontag D, Wang Y. On characterizations of input-to-state stability with respect to compact sets. IFAC Proceedings Volumes 1995; 28: 203-208. 
34. Angeli D, Sontag E. Forward completeness, unboundedness observability, and their Lyapunov characterizations. Systems \& Control Letters 1999; 38: 209-217.

35. Mei W, Efimov D, Ushirobira R, Aleksandrov A. Convergence conditions for Persidskii systems. 2021 European Control Conference (ECC) 2021.

36. Ataeva N. Property of convergence for difference systems. Vestnik SPbSU: Applied mathematics, informatics and control processes 2004; 4: 91-98.

37. Sandberg IW, Willson AN. Some Theorems on Properties of DC Equations of Nonlinear Networks. Bell System Technical Journal 1969; 48: 1-34.

38. Sandberg I. Global inverse function theorems. IEEE Transactions on Circuits and Systems 1980; 27(11): 998-1004.

39. Holt RD, Pickering J. Infectious Disease and Species Coexistence: A Model of Lotka-Volterra Form. The American Naturalist 1985; 126: 196-211.

40. Holland JN, DeAngelis DL, Bronstein JL. Population Dynamics and Mutualism: Functional Responses of Benefits and Costs. The American Naturalist 2002; 159: 231-244.

41. Altafini C. Consensus Problems on Networks With Antagonistic Interactions. IEEE Transactions on Automatic Control 2013; 58(4): 935-946.

42. Baumann F, Lorenz-Spreen P, Sokolov IM, Starnini M. Modeling Echo Chambers and Polarization Dynamics in Social Networks. Phys. Rev. Lett. 2020; 124: 048301.

43. Baumann F, Lorenz-Spreen P, Sokolov IM, Starnini M. Emergence of Polarized Ideological Opinions in Multidimensional Topic Spaces. Phys. Rev. X 2021; 11: 011012. 